\title{
High-energy observations of the state transition of the X-ray nova and black hole candidate XTE J1720-318
}

\author{
M. Cadolle Bel ${ }^{1}$, J. Rodriguez ${ }^{1,2,3}$, P. Sizun ${ }^{1}$, R. Farinelli ${ }^{4}$, M. Del Santo ${ }^{5}$, A. Goldwurm ${ }^{1,6}$, P. Goldoni ${ }^{1,6}$, \\ S. Corbel ${ }^{1,7}$, A. N. Parmar $^{8}$, E. Kuulkers ${ }^{8}$, P. Ubertini ${ }^{5}$, F. Capitanio ${ }^{5}$, J.-P. Roques ${ }^{9}$, F. Frontera ${ }^{4,10}$, \\ L. Amati ${ }^{10}$, and N. J. Westergaard ${ }^{11}$ \\ ${ }^{1}$ Service d'Astrophysique, DAPNIA/DSM/CEA - Saclay, 91191 Gif-sur-Yvette Cedex, France \\ e-mail: mcadolle@cea.fr \\ 2 Integral Science Data Center, Chemin d'Ecogia, 16, 1290 Versoix, Switzerland \\ 3 CNRS FRE 2591, France \\ ${ }^{4}$ Physics Department, University of Ferrara, 44100 Ferrara, Italy \\ 5 IASF-CNR, Via del Fosso del Cavaliere 100, 00133 Roma, Italy \\ ${ }^{6}$ Fédération de Recherche APC, 11 place M. Berthelot, 75231 Paris, France \\ 7 Université Paris VII, France \\ 8 Research and Scientific Support Department, ESA, ESTEC, Keperlaan 1, 2200 AG Noordwijk, The Netherlands \\ 9 Centre d'Étude Spatiale des Rayonnements, CNRS, Toulouse Cedex 4, France \\ ${ }^{10}$ IASF-CNR Section of Bologna, Via P. Gobetti 101, 40129 Bologna, Italy \\ 11 Danish Space Research Institute, Juliane Maries Vej 30, Copenhagen 02100, Denmark \\ Received 14 May 2004 / Accepted 7 July 2004
}

\begin{abstract}
We report the results of extensive high-energy observations of the X-ray transient and black hole candidate XTE J1720-318 performed with INTEGRAL, XMM-Newton and RXTE. The source, which underwent an X-ray outburst in 2003 January, was observed in February in a spectral state dominated by a soft component with a weak high-energy tail. The XMM-Newton data provided a high column density $N_{\mathrm{H}}$ of $1.2 \times 10^{22} \mathrm{~cm}^{-2}$ which suggests that the source lies at the Galactic Centre distance. The simultaneous RXTE and INTEGRAL Target of Opportunity observations allowed us to measure the weak and steep tail, typical of a black-hole binary in the so-called High/Soft State. We then followed the evolution of the source outburst over several months using the INTEGRAL Galactic Centre survey observations. The source became active again at the end of March: it showed a clear transition towards a much harder state, and then decayed to a quiescent state after April. In the hard state, the source was detected up to $200 \mathrm{keV}$ with a power law index of $\sim 1.9$ and a peak luminosity of $\sim 7 \times 10^{36} \mathrm{erg} \mathrm{s}^{-1}$ in the 20-200 keV band, for an assumed distance of $8 \mathrm{kpc}$. We conclude that XTE J1720-318 is indeed a new member of the black hole X-ray novae class which populate our galactic bulge and we discuss its properties in the frame of the spectral models used for transient black hole binaries.
\end{abstract}

Key words. black hole physics - accretion, accretion disks - X-rays: binaries - gamma-rays: observations stars: individual: XTE J1720-318

\section{Introduction}

$\mathrm{X}$-ray Novae $(\mathrm{XN})$, also called soft X-ray transients, are low mass X-ray binaries where a compact object accretes at a very low rate from a late type companion star (Tanaka \& Shibazaki 1996). Although they are usually in a quiescent state (and therefore nearly undetectable), they undergo bright X-ray outbursts, with typical recurrence periods of many years, which last several weeks or even months before the source returns to quiescence. Most of the XN are associated to dynamically proven Black Holes $(\mathrm{BH})$ and indeed the great majority of the known 18 Black Hole Binaries (BHB) as well as of the 22 binary Black Hole Candidates (BHC) are transients (McClintock \& Remillard 2003). Because of large changes in the effective accretion rates that occur during the $\mathrm{XN}$ outbursts and the very hard spectra they usually display, these sources provide powerful probes of the accretion phenomena and radiation processes at work in BH, and are primary targets for high-energy instruments. Indeed, during their outbursts, these sources often undergo changes in their spectral and temporal characteristics; they may pass through the different spectral states observed in BHB (Tanaka \& Lewin 1975; McClintock \& Remillard 2003). The two principal states of BHB are the Low/Hard State (LHS) and the High/Soft State (HSS). In the latter, the emission is dominated by a very soft $(k T \sim 1 \mathrm{keV})$ component generally interpreted as the thermal radiation from an optically thick and geometrically thin accretion disc (Shakura \& Sunyaev 1973). A weak and steep power law may also be present and little variability is observed. In the LHS, the spectrum is rather described by a hard power law with photon index in the range 1.5-2.0 and 
a break around $100 \mathrm{keV}$. The LHS is also characterized by large timing variability. Intermediate states (namely the intermediate state and the very high soft state) are also observed, where both the soft and hard components are present; the source displays a complicated pattern of timing properties. In spite of the recent advances in the characterization of BHB spectral states and modelling of the emission components, the basic mechanisms which generate the state transitions and in particular the origin of the hard component are not yet understood. Detection and broad band studies of new BH systems is therefore essential to acquire better statistics on the phenomenology of the BHB spectral states and on the relations between their emission components.

Since XN probably follow the galactic stellar distribution, they are concentrated in the direction of the bulge of our Galaxy (with a higher density towards the centre). The SIGMA gamma-ray telescope on board the GRANAT satellite, and later the hard X-ray instruments on board Rossi XTE and Beppo SAX discovered and studied several (about 10) BHC XN in the bulge. INTEGRAL, the INTErnational Gamma-Ray Astronomy Laboratory (Winkler et al. 2003) is a European Space Agency observatory launched on 2002 October 17, carrying four instruments: two main gamma-ray instruments, IBIS (Ubertini et al. 2003) and SPI (Vedrenne et al. 2003), and two monitors, JEM-X (Lund et al. 2003) and OMC (Mas-Hesse et al. 2003). The IBIS coded mask instrument is characterised by a wide Field of View (FOV) of $29^{\circ} \times 29^{\circ}\left(9^{\circ} \times 9^{\circ}\right.$ fully coded), a point spread function of $12^{\prime}$ FWHM and it covers the energy range between $20 \mathrm{keV}$ and $8 \mathrm{MeV}$. The SPI telescope works in the range from $20 \mathrm{keV}$ to $8 \mathrm{MeV}$ with a FOV of $31^{\circ}$ diameter ( $16^{\circ}$ fully coded), an angular resolution of $2.5^{\circ}$ $(F W H M)$ and a typical energy resolution of $2.5 \mathrm{keV}$ at $1.3 \mathrm{MeV}$. The JEM-X monitor provides spectra and images with arcminute angular resolution in the 3 to $35 \mathrm{keV}$ band, with a FOV of about $10^{\circ}$ diameter. Thanks to its instruments performances and to the survey program specifically dedicated to the Galactic Centre (GC) region, INTEGRAL allows the detection and study of the hard X-ray emission from BH XN at large distances and at weaker flux levels than before.

XTE J1720-318 was discovered on 2003 January 9 with the All Sky Monitor (ASM) on board RXTE as a transient source undergoing an X-ray nova like outburst (Remillard et al. 2003). The source $1.2-12 \mathrm{keV}$ flux increased to the maximum value of $\sim 430 \mathrm{mCrab}$ in 2 days (see Fig. 1); then its flux started to decay slowly. Follow up observations with the Proportional Counter Array (PCA) on board RXTE showed the presence of a $0.6 \mathrm{keV}$ thermal component and a hard tail. The spectral parameters and the source luminosity were typical of a BH (Markwardt 2003) in the so-called HSS. Soon after, a radio counterpart was identified with the VLA and ATCA radio telescopes (Rupen et al. 2003; O'Brien et al. 2003), leading to the estimate of the most precise position $\alpha_{J 2000}=17^{\mathrm{h}} 19^{\mathrm{m}} 58^{\mathrm{s}} .985$, $\delta_{J 2000}=-31^{\circ} 45^{\prime} 01^{\prime \prime} 109 \pm 00^{\prime} 25$. The detection of its infrared counterpart (Nagata et al. 2003) provided a measure of the extinction which is compatible with a location of XTE J1720-318 at large distance, probably several kpc.

XTE J1720-318 was observed by XMM-Newton, RXTE and INTEGRAL in 2003 February during dedicated Target

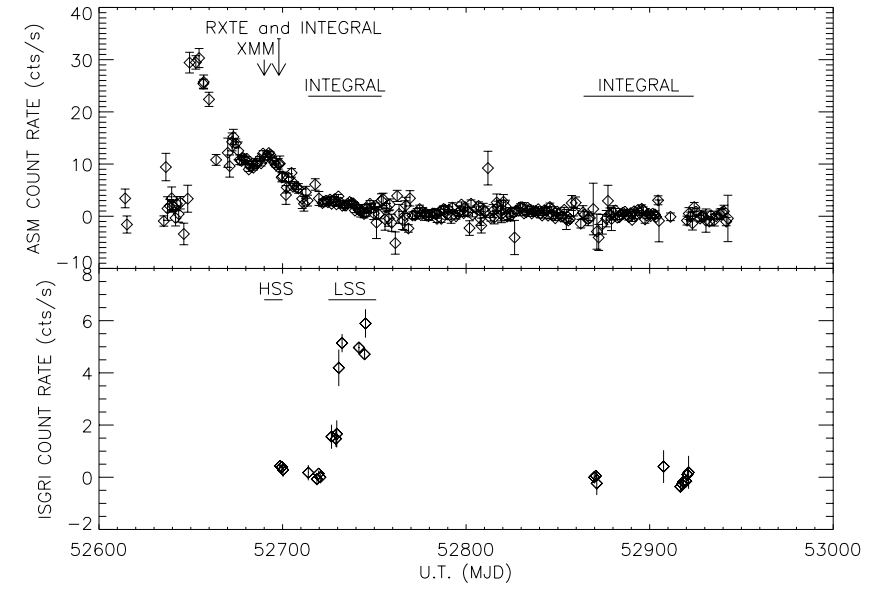

Fig. 1. Top: the RXTE/ASM daily average 1.2-12 keV light curve of XTE J1720-318 from few days before the outburst to 2003 October. The arrows show the dates of the XMM-Newton, RXTE and INTEGRAL observations. The approximate periods of later INTEGRAL observations are indicated by horizontal lines. Universal time is reported in units of MJD. Bottom: the $20-120 \mathrm{keV}$ IBIS/ISGRI light curve of XTE J1720-318 with time bins of 2 days (rev. 46 to 122). The data used to build spectra for HSS and LHS are indicated by horizontal lines.

of Opportunity ( $\mathrm{ToO})$ observations. It was then observed by INTEGRAL during the surveys of the GC region performed in March and April and again from 2003 August to October. We report here the results based from these observations, starting with the description of the available data and of the analysis procedures employed (Sect. 2). We then report the results in Sect. 3 before discussing them in Sect. 4 .

\section{Observations and data reduction}

XTE J1720-318 was observed by XMM-Newton on 2003 February 20, during a public $18.5 \mathrm{ks}$ ToO. Preliminary analysis of these data provided an improved X-ray position of the source (Gonzalez-Riestra et al. 2003), confirming the association with the radio and IR source. One week after, we performed an INTEGRAL ToO observation of XTE J1720-318 which started on 2003 February 28 with a 176 ks exposure. The latter was conducted in coordination with a RXTE ToO observation which lasted about $2 \mathrm{ks}$. The source was further observed during the INTEGRAL Core Program during a series of exposures dedicated to the GC survey, from March 25 to April 19 for a total of $551 \mathrm{ks}$ observing time. Another 275 ks exposure on the source has been accumulated during ToOs on H 1743-322 (Parmar et al. 2003) in 2003 April. The field containing XTE J1720-318 has also been extensively monitored (about $700 \mathrm{ks}$ exposure time) during the second part of the 2003 INTEGRAL GC survey.

The $\log$ of the observations and data used in this work is summarized in Table 1. Figure 1 (top panel) shows the 1.2-12 keV RXTE/ASM light curve of XTE J1720-318 and also indicates the intervals covered by the dedicated XMM-Newton, RXTE and INTEGRAL observations discussed here. 
Table 1. Log of the XTE J1720-318 observations analysed in this paper.

\begin{tabular}{|c|c|c|c|c|}
\hline Spacecraft & $\begin{array}{l}\text { Observation period } \\
\text { Dates (2003) (\# revolution) }\end{array}$ & $\begin{array}{l}\text { Exposure } \\
(\mathrm{ks})\end{array}$ & Instruments & $\begin{array}{l}\text { Observation type } \\
\text { /Mode }\end{array}$ \\
\hline XMM-Newton & $02 / 20$ & 18.5 & EPIC-PN & ToO/Small Window \\
\hline Rossi-XTE & $02 / 28$ & $2 \mathrm{ks}$ & PCA & $\mathrm{ToO}$ \\
\hline INTEGRAL & 02/28-03/02 (46) & 176 & JEM X-2 + IBIS & $\mathrm{ToO}^{a}$ \\
\hline INTEGRAL & $03 / 15-04 / 03(51-57)$ & 361 & IBIS & GCDE \\
\hline INTEGRAL & $04 / 06-04 / 22(58-63)$ & 175 & IBIS + SPI & ToO on $\mathrm{H} 1743-322^{a}$ \\
\hline INTEGRAL & $04 / 12-04 / 19(60-62)$ & 191 & IBIS + SPI & GCDE \\
\hline INTEGRAL & $08 / 02-10 / 16(103-122)$ & 700 & IBIS & GCDE \\
\hline
\end{tabular}

Notes: ${ }^{a} 5 \times 5$ dithering pattern around the target.

\subsection{XMM-Newton data analysis}

We present here the data taken with the EPIC-PN camera on board XMM-Newton. The PN camera was operating in Small Window mode. We processed the data using the Scientific Analysis System v5.4.1 and the calibration files updated at the end of 2003 March. We first filtered our data for background flares. Since XTE J1720-318 was bright at the date of the observation (resulting in a strong pile up in the PN camera), we adopted the selection criteria suggested by Guainazzi (2001) to obtain the source spectrum. We extracted the single events from an annulus with an internal radius of $15^{\prime \prime}$, and an outer radius of 29" around the position of XTE J1720-318. As we only used single events in this corona, the effective exposure time of the extracted spectrum was about $6 \mathrm{ks}$.

We obtained the background spectrum from a sky region far from the source and we built the response matrix (RMF) and ancillary response (ARF) files consistent with the selections. Adding 2.5\% systematics, the resultant spectrum was then fitted with XSPEC v11.3.0 (Arnaud 1996) between 0.7 and $11 \mathrm{keV}$.

\subsection{Rossi XTE data analysis}

We reduced and analysed the RXTE data with the LHEASOFT package v5.3. We reduced the data from the PCA following the standard methods explained in the ABC of RXTE and the cook book. The good time intervals (GTI) were defined when the satellite elevation was $>10^{\circ}$ above the Earth's limb, and the offset pointing $<0.02^{\circ}$. We also chose to retain the data taken when most of the Proportional Counter Units (PCU) were turned on (a maximum of 5 here). We extracted the spectra from the standard 2 data, from the top layer of each PCU. Background spectra were produced with pcabackest v3.0, using the latest calibration files available for bright sources. The RMF and ARF were generated with pcarsp v8.0. Due to uncertainties in the PCA RMF, we included some systematic errors in the spectra. To estimate the level of those systematics, we reduced and analysed a contemporary Crab observation. To obtain a reduced $\chi^{2}$ of 1 when fitting the Crab spectra, we set the level of systematics as follows: $0.6 \%$ between 2 and $8 \mathrm{keV}$ and $0.4 \%$ above $8 \mathrm{keV}$. We fitted the spectra between $3-25 \mathrm{keV}$ for the PCA. We also processed HEXTE data but, due to the poor statistics, we did not include the few HEXTE data points in the analysis.
For the timing analysis, we extracted $16 \mathrm{~s}$ resolution PCA light curves from standard 2 data, using all PCUs and all layers, between 2 and $20 \mathrm{keV}$ (absolute channels 5-49), and corrected them for background. We extracted high temporal resolution light curves from the event mode data with a nominal resolution of $2^{-13} \mathrm{~s}$ (i.e. $\sim 125 \mu \mathrm{s}$ ) rebinned to $1 \mathrm{~ms}$ during the extraction processes. Three such light curves were extracted between absolute channels 5-49 (2-20 keV epoch 5), 17-49 (7-20 keV) and 24-49 (10-20 keV).

\subsection{INTEGRAL data analysis}

An INTEGRAL observation is made of several pointings (science windows, hereafter SCW) each having exposure time lasting from 1800 to $3600 \mathrm{~s}$ and following a special pattern on the plane of the sky (Courvoisier et al. 2003). Except for the $5 \times 5$ dithering mode for ToOs, the entire GC region was observed in the framework of the Galactic Centre Deep Exposure (GCDE) program (Winkler 2001). Deep exposures in the GC radian $( \pm 30 \mathrm{deg}$ in longitude, $\pm 20 \mathrm{deg}$ in latitude centred at $l=0, b=0$ ) are obtained with a set of individual pointings lasting $30 \mathrm{~min}$ each on a regular pointing grid.

All the INTEGRAL instruments were operating simultaneously. We describe here mainly results obtained from the data recorded with the ISGRI detector (Lebrun et al. 2003) of the IBIS telescope covering the spectral range from 20 to $800 \mathrm{keV}$. For the first observation set, when the source was very soft, we also present data from the JEM-X instrument. The IBIS data have been reduced with the Offline Scientific Analysis (OSA) v3.0 delivered in December 2003 to produce images and extract spectra for each SCW (Goldwurm et al. 2003). We selected SCW for which the source was within $8^{\circ}$ from the telescope axis. For the spectral analysis, we used a 12 linearly rebinned channel RMF and the associated recently corrected ARF (P. Laurent, 2003 December, private communication). The resultant spectrum was fitted between 20 and $600 \mathrm{keV}$, but above $200 \mathrm{keV}$ the source is not always significantly detected and below $20 \mathrm{keV}$ systematic uncertainties are still very high. Systematics errors at level of $8 \%$ (see Sect. 3.3) were applied in the spectral fits to account for the residual effects of the response matrix (Goldwurm et al. 2003). For the image analysis, the background derived from empty fields was subtracted before deconvolution and we used a catalog of about 41 sources to analyse the images. The total amount of IBIS data we 
processed was equivalent to about $1700 \mathrm{ks}$ of exposure time, however due to selections performed and the fact that the source was very often off-axis, the effective exposure time is reduced to $652 \mathrm{ks}$.

We reduced the JEM-X data with OSA v3.0. Only the JEM-X2 monitor was operated during our observation. Because of uncertainties in the RMF for high off-axis angles, we selected only SCW where the source was closest to the centre of the field of view (i.e. $<3^{\circ}$ from the telescope axis). The energy channels were also rebinned so as to have a $\sigma>3$. When all these conditions hold, we extracted the spectra for an effective exposure time of $21 \mathrm{ks}$. We fitted the resultant averaged spectrum between 3.5 and $26.5 \mathrm{keV}$, with the standard RMF and ARF.

Concerning SPI, the data obtained during revolutions (hereafter, rev.) 58 and 60 to 63 were reduced using the Spi_science_analysis_2 script (Knödlseder 2004) available in OSA v3.0 (February 2004). Images were then extracted between 20 and $40 \mathrm{keV}$ to build a catalogue of sources. Spectra were finally extracted for each data set with 50 logarithmic bins in the $20-1000 \mathrm{keV}$ energy range, using Spiros (Skinner \& Connell 2003). We used the imaging results from the IBIS/ISGRI telescope to determine the active sources of the region in order to account for their contribution in the SPI spectral extraction. The latter was performed with a background model derived from the evolution of the saturated count rates in the Ge detectors while the ratios between detectors were left free in the analysis. A few SCW with a bad $\chi^{2}$ were excluded. Extracted with Image Response Files v15, the resulting spectra were fitted with the RMF delivered in 2004 February.

\section{Results of the analysis}

\subsection{The INTEGRAL detection of the XTE J1720-318 state transition}

The 20-120 keV IBIS/ISGRI light curve of XTE J1720-318 from the whole set of the 2003 INTEGRAL data is shown in Fig. 1 (lower panel) on the same scale of the ASM light curve. During the INTEGRAL ToO observation of February 28 (rev. 46, MJD 52699), the source was detected at a very low flux level above $20 \mathrm{keV}$ and 10 days later (i.e. between March 9 and 20), it had decreased below the detection level. Starting from March 25 (MJD 52724, rev. 54), the source appeared to brighten in the INTEGRAL/IBIS energy band. Since a similar behaviour was not seen in the ASM light curve (see Fig. 1, top panel), Goldoni et al. (2003) proposed that the source was entering a hard state. Figure 2 shows the details of this hard flare. The $20-80 \mathrm{keV}$ flux was at the beginning around $2 \mathrm{cts} \mathrm{s}^{-1}(\sim 11 \mathrm{mCrab})$ and increased to a maximum level of $6.25 \mathrm{cts}^{-1}$ ( $\left.\sim 34.5 \mathrm{mCrab}\right)$ on April 6 (rev. 58, MJD 52737). After this, the flux gradually decreased to the value of $4 \mathrm{cts} \mathrm{s}^{-1}$ (rev. 63, MJD 52751). When the INTEGRAL GC survey included the source again in the IBIS FOV in mid August 2003, the transient was not detected and remained below the IBIS/ISGRI detection level for the rest of 2003 (Fig. 1). The derived $3 \sigma$ upper limit on XTE J1720-318 flux during the mid-August

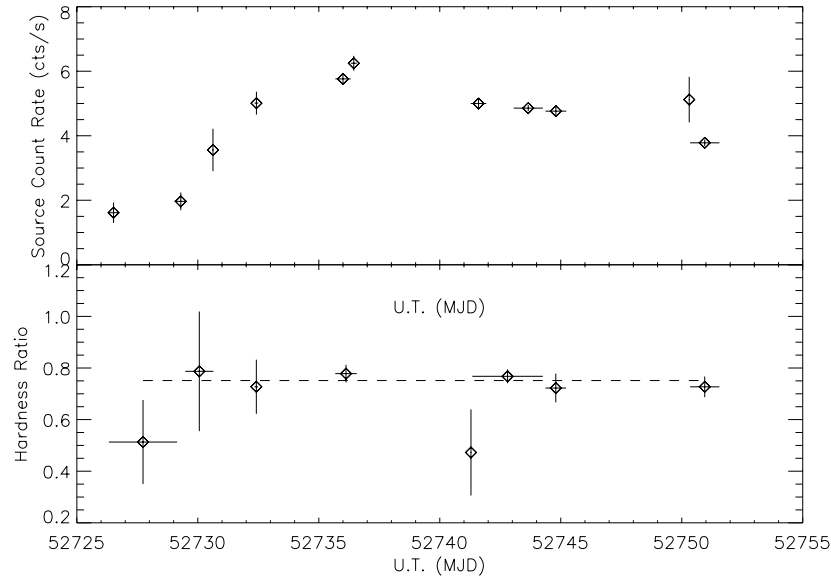

Fig. 2. Top: IBIS/ISGRI $20-80 \mathrm{keV}$ light curve with time bins $\sim 1$ day during the XTE J1720-318 hard flare (rev. 55 to 63). Bottom: corresponding hardness ratio, defined as the ratio between the source count rate in the $40-80 \mathrm{keV}$ band and in the $20-40 \mathrm{keV}$ band with time bins of 3 days. The dashed line represents the average HR.

observations ( MJD 52869, exposure of $271 \mathrm{ks}$ ) is $1.5 \mathrm{mCrab}$ between $20-80 \mathrm{keV}$.

Figure 2 shows the hardness ratio (HR) measured during the observed increase of the source high energy flux. There is no significant variation in the HR around its mean value of 0.75 , only a slight indication of a softer HR $(\sim 0.5)$ at the beginning of the flare. We therefore used the whole data of this hard flare to build up an average spectrum (see Sect. 3.3.2). Moreover, we could add the data taken with SPI, which provide us significant points during this period at high energies. We also analysed JEM-X data taken during the hard flare, but due to the fact that the source was often at large off-axis angles and very faint below $20 \mathrm{keV}$, the derived data points were not significant; therefore, we did not use them in the analysis.

Assuming an exponential shape for both the rise and the decay phases of the hard flare we obtained, using the IBIS/ISGRI data points of Fig. 1 (lower panel), time constants of 13 days (rise) and 48 days (decay) respectively. Since we used the August upper limits for this estimate, the characteristic decay time we derived is therefore only an upper limit. However, the hard flare timescales appear comparable to the main outburst (the characteristic decay time is 60 days) even if the peak broad-band X-ray luminosity remains well below the peak luminosity of the main outburst (see Sect. 3.3).

In the combined IBIS/ISGRI images obtained during the hard outburst (data from rev. 58 to 61), XTE J1720-318 is detected at $94 \sigma$ in the $20-60 \mathrm{keV}$ range (Fig. 3). The best position found with IBIS from the $20-60 \mathrm{keV}$ image is $\alpha_{J 2000}=$ $17^{\mathrm{h}} 19^{\mathrm{m}} 58^{\mathrm{s}} .7, \delta_{J 2000}=-31^{\circ} 44^{\prime} 43^{\prime \prime} .7$ with an accuracy of 0.45 at $90 \%$ confidence level (Gros et al. 2003). This position is consistent with the most precise position of XTE J1720-318 derived from radio data since the offset is only $17^{\prime \prime} .7$. The high-energy source is therefore unambiguously associated to the transient. 


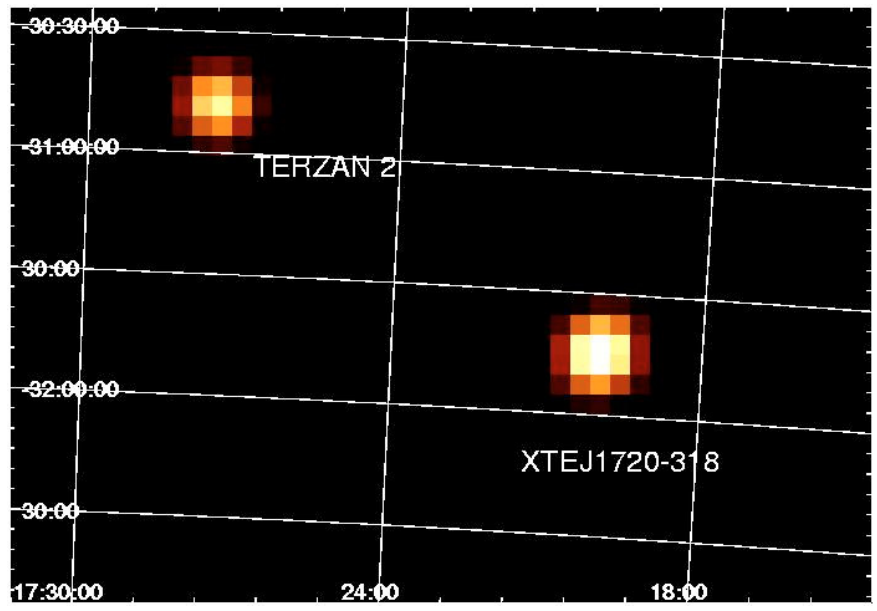

Fig. 3. The IBIS/ISGRI reconstructed sky image of the region around XTE J1720-318 in the 20-60 keV band (rev. 58 to 61). XTE J1720-318 appears at a significance level of $94 \sigma$ over the background. The other source in the image is the hard X-ray burster located in the globular cluster Terzan 2.

\subsection{XTE J1720-318 timing variability during the high/soft state}

The XMM-Newton and the INTEGRAL/RXTE observations of February 2003 caught the source in a very soft state (HSS). The source appeared bright at low energies, with a dailyaveraged flux between 100 and $140 \mathrm{mCrab}$ in the $1.2-12 \mathrm{keV}$ band. The JEM-X and PCA instruments detected the source at very high significance and we could derive significant spectra up to $20 \mathrm{keV}$ (Fig. 6). However, the high-energy emission was quite weak. IBIS detected the source at a level of $0.4 \pm$ $0.07 \mathrm{cts} \mathrm{s}^{-1}(\sim 2.1 \mathrm{mCrab})$ in the $20-120 \mathrm{keV}$ band with a signal of $6 \sigma$, providing only few data points at energies higher than $50 \mathrm{keV}$. As RXTE/HEXTE provided low significant data points at energy $\geq 20 \mathrm{keV}$, we did not include them in the spectra (described in Sect. 3.3.1).

The PCA 2-20 keV light curves are shown in Fig. 4. XTE J1720-318 shows some variations around a mean value of $\sim 750 \mathrm{cts} \mathrm{s}^{-1}$ in the first set, and $721 \mathrm{cts} \mathrm{s}^{-1}$ in the second set. A slight decrease is visible from the first observation to the second (Fig. 4). The light curve of the latter is characterised by an increase of the flux to $\sim 750 \mathrm{cts} \mathrm{s}^{-1}$, during a $\sim 200 \mathrm{~s}$ small flare. We produced Power Density Spectra in the 3 energy ranges described in Sect. 2.2 with POWSPEC v1.0. These energy dependant PDS were produced on interval length of $16 \mathrm{~s}$ between $62.5 \mathrm{mHz}$ and $500 \mathrm{~Hz}$. All the intervals (from the 2 data sets) were averaged in a single frame, and a geometrical rebinning has been applied. The 2-20 keV Leahy normalised PDS is flat (Fig. 4, lower panel). The best-fit model is a constant value of $1.993 \pm 0.004$ (at the $90 \%$ confidence level) with a $\chi^{2}$ of 87.6 (105 degrees of freedom, hereafter d.o.f.). This value is compatible with the expected Leahy normalised value of 2 for purely Poisson noise (white noise). At higher energy the PDSs are also flat. The $3 \sigma$ upper limits on the $2-20 \mathrm{keV}$ fractional level of variability is $\sim 6.7 \%$. In the higher energy ranges, the upper limit is rather high and meaningless due to the low statistics of the source.
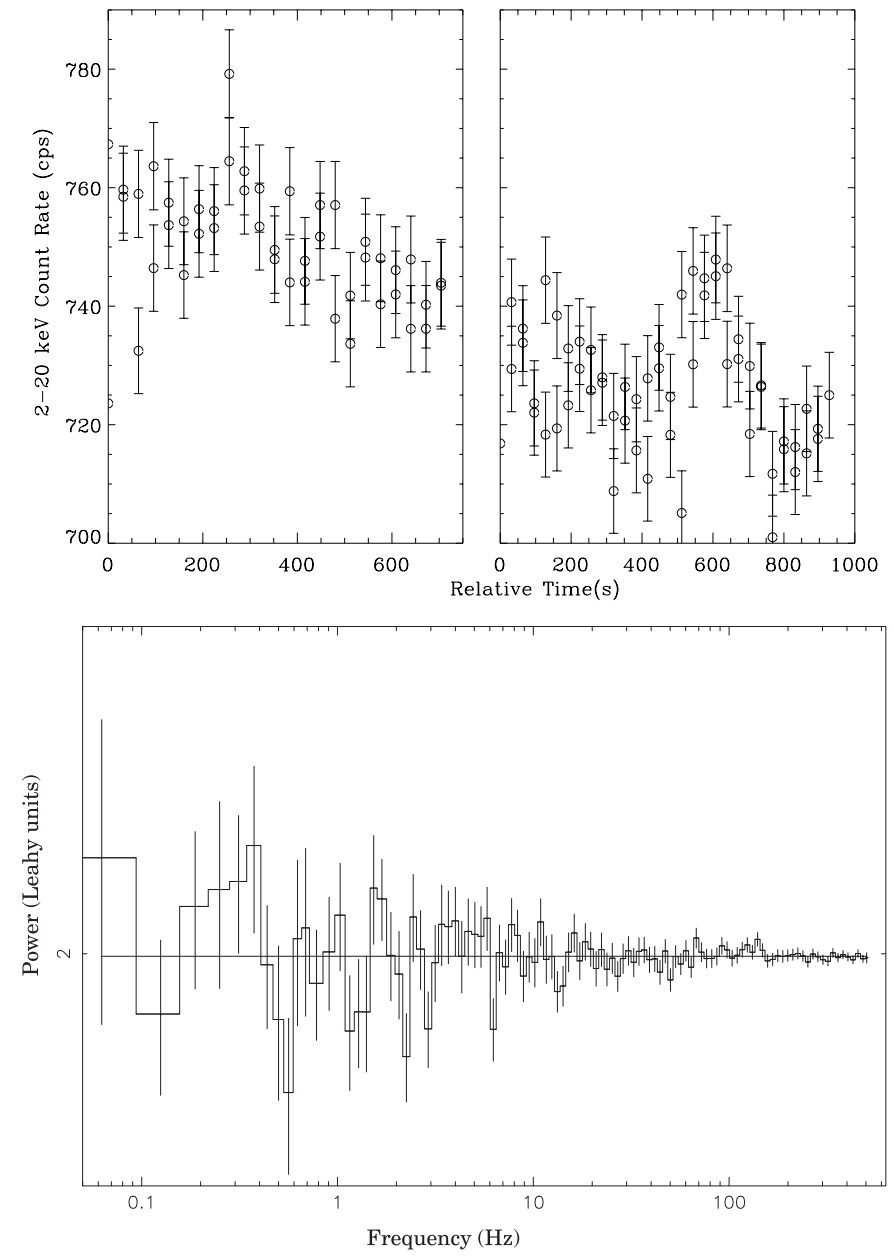

Fig. 4. Top: 2-20 keV RXTE/PCA light curves in counts per second, covering the two data sets. For both, relative time 0 refers to the beginning of the GTIs: time 0 corresponds to MJD $=52698.506$ (28) (left panel) and to MJD = 52699.487(31) (right pannel). The time sampling is $16 \mathrm{~s}$. Bottom: $2-20 \mathrm{keV}$ PDS of the combined RXTE/PCA data sets. The best-fit (constant) is superimposed as a line.

\subsection{Spectral results}

\subsubsection{The high/soft state spectrum}

We have fitted the XMM-Newton EPIC-PN data with a model composed of an absorbed multi-colour black-body disc (MCD, Mitsuda et al. 1984) plus a power law. A single absorbed MCD alone leads to a poor fit $\left(\chi^{2}=1349\right.$ for 1064 d.o.f. $)$, as does a single absorbed power law $\left(\chi^{2}=14825\right.$ for 1064 d.o.f.). The best-fit parameters derived from our analysis are given in Table 2. We obtained for $N_{\mathrm{H}}=(1.24 \pm 0.02) \times 10^{22} \mathrm{~cm}^{-2}$. The unabsorbed flux in the $0.7-10 \mathrm{keV}$ range is $6.43 \times$ $10^{-9} \mathrm{erg} \mathrm{cm}^{-2} \mathrm{~s}^{-1}$. Assuming a distance of $8 \mathrm{kpc}$ (see discussion), we derive a $0.7-10 \mathrm{keV}$ unabsorbed) luminosity of $4.9 \times$ $10^{37} \mathrm{erg} \mathrm{s}^{-1}$. The disc component accounts for more than $85 \%$ of the total $2-100 \mathrm{keV}$ luminosity. If we assume a line of sight inclination angle $(\theta)$ of $60^{\circ}$, we find, from the disc normalisation, an inner disc radius of $48.7 \pm 0.5 \mathrm{~km}$. Figure 5 shows the resultant $\mathrm{EF}(\mathrm{E})$ spectrum and its best-fit.

We have applied the same absorbed MCD plus a power law model to a simultaneous fit of the RXTE/PCA, 
Table 2. XTE J1720-318 best-fit spectral parameters (with 90\% confidence level errors) for the XMM-Newton and the RXTE/INTEGRAL ToOs of February.

\begin{tabular}{lllllll}
\hline \hline Satellite & $\begin{array}{l}\text { Date } \\
(2003)\end{array}$ & $\begin{array}{l}\text { Photon } \\
\text { index }\end{array}$ & $\begin{array}{l}\text { Disc tempe- } \\
\text { rature (keV) }\end{array}$ & $\begin{array}{l}\text { Disc norma- } \\
\text { lisation }\end{array}$ & $\begin{array}{l}\chi_{\text {red }}^{2} \\
\text { (d.o.f. })\end{array}$ & $\begin{array}{l}\text { Flux } \\
\left(\times 10^{-9} \mathrm{erg} \mathrm{cm}^{-2} \mathrm{~s}^{-1}\right)\end{array}$ \\
\hline XMM-Newton & $02 / 20$ & $2.81_{-0.66}^{+0.60}$ & $0.67 \pm 0.01$ & $1855_{-43}^{+37}$ & $0.84(1062)$ & 2.36 \\
RXTE+INTEGRAL & $02 / 28-03 / 02$ & $2.72_{-0.34}^{+0.29}$ & $0.59 \pm 0.01$ & $5647_{-404}^{+338}$ & $0.97(72)$ & 3.25 \\
\hline
\end{tabular}

Notes: ${ }^{a}$ Disc normalisation $K$ is given by $K=\left(\frac{R}{D}\right)^{2} \times \cos \theta$ where $R$ is the inner disc radius in units of km, $D$ is the distance to the source in units of $10 \mathrm{kpc}$ and $\theta$ the inclination angle of the disc.

${ }^{b}$ Unabsorbed $2-100 \mathrm{keV}$ flux.

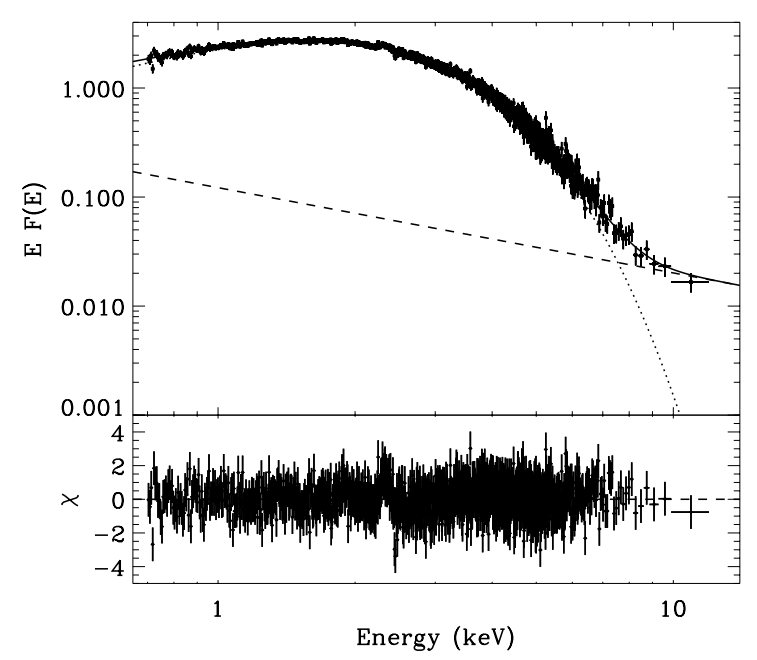

Fig. 5. XMM-Newton/EPIC-PN unabsorbed EF(E) spectrum of XTE J1720-318 (units $\mathrm{keV} \mathrm{cm}^{-2} \mathrm{~s}^{-1}$ ) along with the best-fit model MCD plus power law. Dotted: MCD. Dashed: power law. Thick: total model. Residuals (in $\sigma$ units) are also shown.

INTEGRAL/JEM-X and INTEGRAL/IBIS data taken about 8 days later. We obtained the best-fit parameters reported in Table 2. To account for uncertainties in relative instruments calibrations, we let a multiplicative constant free to vary in the fit of the different data sets. Taking the RXTE/PCA spectrum as the reference, the derived constants are all found very close to 1 for each instrument. As RXTE and JEM-X are not suited to determine interstellar absorption (energy lower boundary is $\sim 3 \mathrm{keV}$ ), we fixed $N_{\mathrm{H}}$ to the value obtained from the XMM-Newton fits. We also added a gaussian function at the iron fluorescent line energies to account for a feature present in the RXTE data. The line centroid was found to be $6.45_{-0.35}^{+0.16} \mathrm{keV}$ with an equivalent width $(E W)$ of $572_{-178}^{+307} \mathrm{eV}$. However, this line was not present in the data obtained with XMM-Newton. To check the reality of this line, we reperformed the fit of the EPIC PN spectrum by adding to the best fit continuum model an iron line at a fixed energy and width equal to the ones found from the RXTE data $(F W H M=1.6 \mathrm{keV})$. We obtained an upper limit for such a line of $250 \mathrm{eV} \mathrm{EW}$ at the $90 \%$ confidence level. For a narrow line at the same energy, we obtained an upper limit of $75 \mathrm{eV} \mathrm{EW}$. This upper limit suggests that the line seen with RXTE is probably due to an incorrect background subtraction and not to XTE J1720-318. Indeed a contamination by the galactic ridge emission (Revnivtsev 2003) cannot be excluded even if the line should be rather centered at 6.7 with a narrower width. With a line centroid fixed to this energy, we obtained from the RXTE spectrum a line width of $0.59{ }_{-0.21}^{+0.06} \mathrm{keV}$ and EW of $456_{-136}^{+117} \mathrm{eV}$. The residuals are slightly worse around the 6.4-6.7 range but they do not exclude such a line contamination. Detailed analysis of other RXTE/PCA data of XTE J1720-318 during the outburst will probably clarify this issue. For this reason, we did not included the line for the fit of the INTEGRAL data. In spite of the low significance level of the detection, the IBIS/ISGRI data allow us to study the source up to higher energies because of the higher sensitivity of ISGRI and the longer exposure time. The 3-200 keV count spectrum and the derived best-fit model are shown in Fig. 6. Figure 7 (red) shows the unfolded EF(E) spectrum with its best-fit model. Note that above $\sim 100 \mathrm{keV}$, the source is not significantly detected.

The disc inner radius (with the same assumptions on distance and viewing angle as above) is $85_{-4}^{+2} \mathrm{~km}$ and the disc flux luminosity contributes to $93 \%$ of the unabsorbed $2-100 \mathrm{keV}$ luminosity. Indeed, there is a slight evolution between the XMM-Newton derived disc parameters (radius and temperature) and the same parameters found one week later by RXTE and INTEGRAL while the power law slope did not change. According to the strengths of the soft component and the value of the power law photon index, we found that the source was clearly in a HSS, where the thermal component from the accretion disc dominates and the high energy tail is very weak. We have also fitted the data with a bulk motion comptonisation model (Shrader \& Titarchuk 1999), often used to model the spectra of BH in HSS. The test gave us an acceptable fit with $\chi_{v}^{2}$ of 1.36 for 78 d.o.f. The derived temperature of thermal photon source is $0.52 \pm 0.01 \mathrm{keV}$, the energy spectral in$\operatorname{dex} 1.8_{-0.4}^{+0.2}$ and the log A parameter $-1.6_{-0.2}^{+0.1}$, compatible with the parameters obtained from the MCD plus power law fit. The spectra taken during the last week of 2003 February are therefore all consistent with the hypothesis that XTE J1720-318 is a BH XN in HSS.

\subsubsection{The low/hard state spectrum}

As discussed above, IBIS data from rev. 55 to 63 , during the hard flare, are consistent with one another (i.e. no variation of HR) and can be summed to derive the average spectrum and its best-fit model reported in Fig. 7 (blue). We fitted this spectrum with a simple power law model between 20 and $600 \mathrm{keV}$. We also used the count spectrum derived from SPI data of rev. 58 to 61 in order to make a simultaneous fit. Due to the 


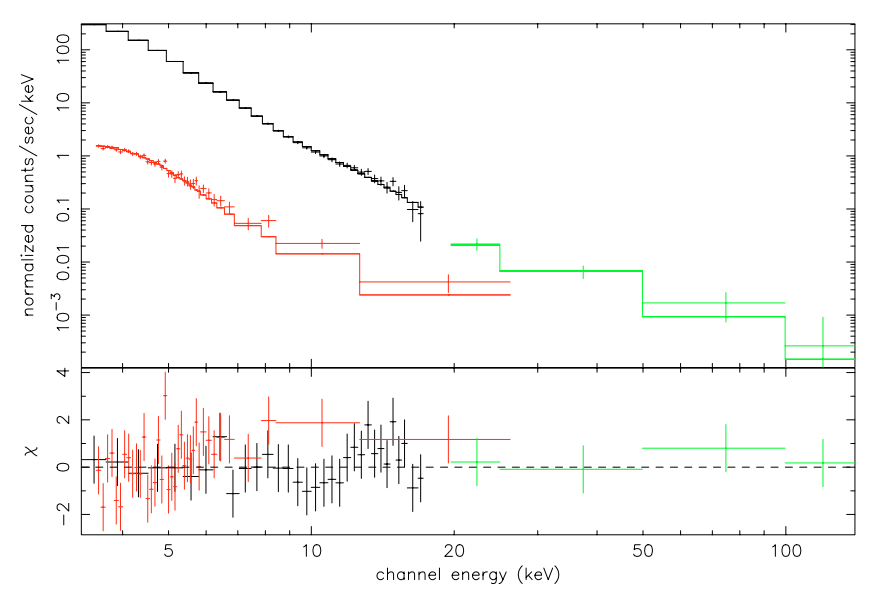

Fig. 6. Joint RXTE/PCA (black), INTEGRAL/JEMX-2 (red) and INTEGRAL/IBIS (green) spectra of XTE J1720-318 during the observations of end 2003 February. The best-fit model, an absorbed MCD plus a power law, is over plotted as a solid line to the data. Residuals (in $\sigma$ units) are also shown.

presence of high background structures, SPI data from rev. 62 to 63 were not included in the spectra. The SPI data points were binned so as to have a level of $3 \sigma$ per bin or 4 bins together at least. To account for uncertainties in relative instruments calibrations, we let a multiplicative constant vary in the fit. Taking the IBIS spectrum as the reference (constant equal to 1), the multiplication factor returned for SPI during the fit is 1.28. At $90 \%$ confidence level, the best-fit photon index returned from the fits is $1.9 \pm 0.1$ with a reduced $\chi^{2}$ of 1.60 ( 22 d.o.f.), which reveals that the spectrum of XTE J1720-318 is much harder than observed in February. In addition to the power law model, we fitted the data set with a comptonisation model (Sunyaev \& Titarchuck 1980): the XSPEC compst model. The derived parameters are $43{ }_{-11}^{+32} \mathrm{keV}$ for the temperature and $2.7 \pm 0.9$ for the optical depth, with a reduced $\chi^{2}$ of 1.27 (21 d.o.f.). Similarly we fitted the spectrum with a comptonisation model (the XSPEC comptt model) which includes relativistic effects, estimates a larger range of parameters and includes the seed soft photon energy (Titarchuk 1994). We obtained, with a seed photon temperature frozen to $k T_{\mathrm{bb}}=0.6 \mathrm{keV}$ and a spherical geometry, a plasma temperature of $k T=57 \pm 29 \mathrm{keV}$ and an optical depth $\tau=2 \pm 1$ with a reduced $\chi^{2}$ of 1.19 (21 d.o.f.). We also tested the Putanen \& Svensson (1996) comptonisation model (the XSPEC compps model) proper for very hot plasmas: we obtained $k T=430 \pm 110 \mathrm{keV}$ and $\tau$ equal to $0.11 \pm$ 0.11 , for a fixed seed photon $k T_{\mathrm{bb}}=0.45$, with a reduced $\chi^{2}$ of 1.33. Since the latter models gave slightly better fit than the single power law, we have performed an additional test to see if a break in the power law would be statistically significant. We fitted a cut-off power law and derived the difference between the absolute $\chi^{2}$ with the $\chi^{2}$ of the single power law. We restricted the fit to the data up to $300 \mathrm{keV}$ to avoid the use of upper limits, and we obtained a $\Delta \chi^{2}$ of 5.8; for a $\chi^{2}$ distribution with 1 d.o.f., this value represents a probability of $95 \%$ that the new component is significant. Even if the test is not fully conclusive, a cut-off in the model with a typical folding energy of approximately $120 \mathrm{keV}$ clearly improves the fit and better describes the available data. We note that the derived thermal

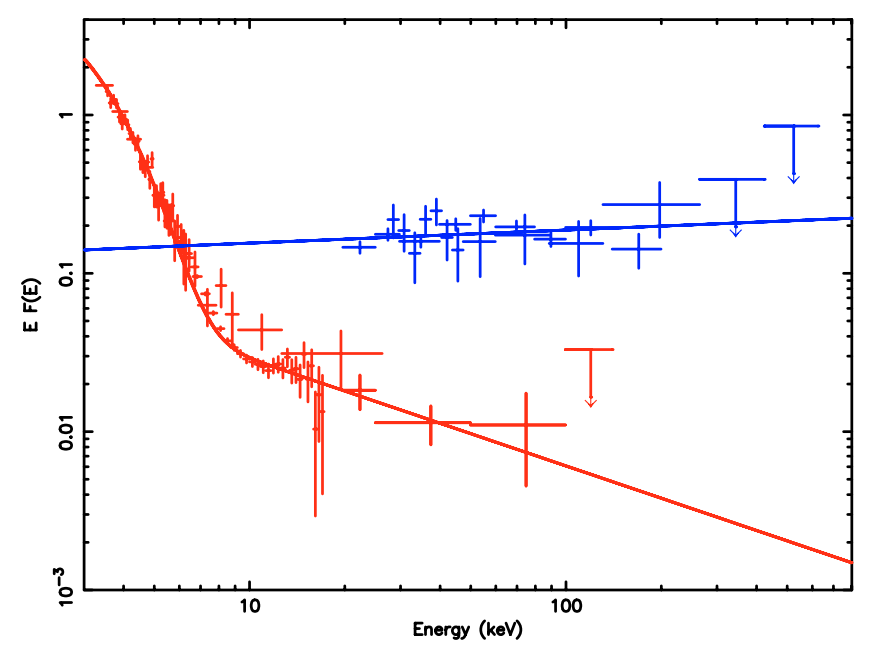

Fig. 7. Unabsorbed EF(E) spectrum of XTE J1720-318 (units of $\mathrm{keV} \mathrm{cm} \mathrm{cm}^{-2} \mathrm{~s}^{-1}$ ) along with the best-fit model: MCD plus power law for the HSS (red) and a power law for the LHS (blue) with IBIS/ISGRI and SPI 20-600 keV data (rev. 55 to 63). Upper limits are shown at $3 \sigma$.

comptonisation parameters for XTE J1720-318 during the hard flare (or the best-fit power law index and cut-off energy) are very much consistent with those found in BHB in the so-called LHS.

\section{Discussion}

The high equivalent absorption column density derived from the XMM-Newton data suggests that XTE J1720-318 lies at the GC distance or even further. This would place the source in the galactic bulge and we will, therefore, assume a distance to the source of $8 \mathrm{kpc}$.

When observed with XMM-Newton, about 40 days after the outburst peak, XTE J1720-318 was clearly in a HSS, characterized by a strong soft (thermal) component, well modelled by a MCD model with an inner disc temperature of $k T \sim$ $0.7 \mathrm{keV}$, and a weak power law tail (Fig. 5). The source was found in HSS also at the end of February (Figs. 6 and 7) when we could measure, with higher precision, using INTEGRAL and RXTE simultaneous observations, the power law index of 2.7. In both observations, the disc component accounted for more than $85 \%$ of the unabsorbed $2-100 \mathrm{keV}$ source luminosity, estimated at the end of February at $2.5 \times 10^{37} \mathrm{erg} \mathrm{s}^{-1}$. We estimated the bolometric luminosity from the best fit spectrum by extending the flux computation at $0.01 \mathrm{keV}$. We obtained for the XMM observation a value of $1.4 \times 10^{38} \mathrm{erg} \mathrm{s}^{-1}$. Even for a small $5 M_{\odot} \mathrm{BH}$ (see discussion below), this bolometric luminosity is below the Eddington luminosity which is $6.5 \times$ $10^{38} \mathrm{erg} \mathrm{s}^{-1}$ for such a BH mass. Similar results are obtained one week later with RXTE/INTEGRAL observations: the accretion rate is sub-Eddington.

Besides, no line emission was observed with XMM-Newton and we could determine an upper limit to the $E W$ of 75 and $144 \mathrm{eV}$ for narrow lines at 6.4 and $6.7 \mathrm{keV}$ respectively. The upper limits for broad lines are less constraining. As discussed in Sect. 3.3.1, we consider unlikely that the relatively strong iron line $(\sim 570 \mathrm{eV})$ we detected with RXTE 
about 10 days later can be due to the source since we obtained an upper limit of $250 \mathrm{eV}$ with XMM data and we did not observe large spectral changes between the two observations. The XMM-Newton narrow line upper limit is below the strong $E W \mathrm{~s}(>150 \mathrm{eV})$ of lines observed in certain $\mathrm{BH}$ systems and attributed to fluorescence produced by reflection of hard $\mathrm{X}$-rays from the accretion disc (e.g. Miller et al. 2001).

However, these line are often broadened by relativistic effects and in this case our data are less constraining. For the parameters of the emission line (centroid at $6.2 \mathrm{keV}$ and $F W H M$ of $2.4 \mathrm{keV}$ ) reported by Markwardt (2003) from an RXTE observation of XTE J1720-318 performed during the main outburst peak, we in fact obtained upper limits of only $290 \mathrm{eV}$, while the RXTE measured EW was $95 \mathrm{eV}$. Only slight differences in the spectral parameters of the soft component were measured between the INTEGRAL/RXTE observations and the XMM-Newton one (i.e. a lower temperature and a larger inner disc radius). The inner radius derived from the normalisation of the MCD model for XTE J1720-318 (for the assumed distance and inclination angle) is compatible with values of the radius of the innermost stable circular orbit around a Schwarzschild BH with mass of $\sim 5 M_{\odot}$. During the outburst decay of $\mathrm{BH} \mathrm{XN}$, this parameter is observed to remain approximately constant if the source stays in the HSS (McClintock \& Remillard 2003; Ebisawa et al. 1991). The variation observed in XTE J1720-318 could indicate that the disc was receding during the decay phase, but it is more probably linked to a specific variation of the normalisation during a secondary flare. Indeed the XMM-Newton observation took place right at the maximum of a weak secondary peak which occurred in the decay phase (see Fig. 1, top panel) and which was also observed in infrared (Nagata et al. 2003). In anycase, these differences could also be due at least in part to cross calibration uncertainties between the instruments. The lack of significant rapid variability that we have found from the study of the PDS using RXTE data, is also compatible with the source being in the HSS. In this state in fact, the fractional time variability is in general lower than 5-10\%. From our data, considering the results previously reported by Markwardt et al. (2003) and the RXTE/ASM light curve which steadily decreased during 2003, we can conclude that the source did not change spectral state during the decay phase which started after the main outburst peak and lasted until about mid March.

A dramatic change in the source behaviour was instead observed with INTEGRAL towards the end of March. After the source had decreased below the INTEGRAL detection level, we observed the rise of the high-energy component about 75 days after the main outburst peak, giving rise to a secondary outburst which we could observe for about 25 days. Since such increase was not seen in the RXTE/ASM count rate, and as we did not have significant signal in the JEM-X data, we conclude that the source underwent a spectral transition towards the LHS. The 20-200 keV unabsorbed luminosity increased in about 10 days from below the INTEGRAL detection level to a value of $7 \times 10^{36} \mathrm{erg} \mathrm{s}^{-1}$. Then it started to decrease with timescale between 10 days and 50 days.

During this secondary outburst, the $>20 \mathrm{keV}$ spectrum was hard and well described by a power law photon index of 1.9 or a thermal comptonisation model with a (weakly constrained) plasma temperature of $43 \mathrm{keV}$ and an optical depth of 2.7. The spectral break seems probable, but no firm conclusion is possible due to the low significance of the derived source spectrum at high energies. The derived best-fit parameters (both the power law slope and break and the temperature and depth of the comptonising plasma) are however compatible with those typically found in BHB in the LHS. Assuming that the power law extends at low energies without any additional contribution of a soft component, we estimated an average unabsorbed $2-200 \mathrm{keV}$ luminosity of $\sim 9.4 \times 10^{36} \mathrm{erg} \mathrm{s}^{-1}$. The bolometric luminosity is estimated to $4.3 \times 10^{37} \mathrm{erg} \mathrm{s}^{-1}$ and shows again that the source was in a sub-Eddington regime, even for a low mass BH. While this secondary outburst did not reach the luminosity of the main one, it is clear that the transition is not simply due to spectral pivoting as observed in Cygnus X-1.

The high peak luminosity, the fast rise and slow decay time scales, the HSS and the secondary outburst with transition to a LHS with spectral parameters typically observed in other (dynamically confirmed) BH transients, like e.g. XTE J1550-564 (Sobczak et al. 2000; Rodriguez et al. 2003) or GRO J1655-40 (Sobczak et al. 1999, see also McClintock \& Remillard 2003), clearly show that XTE J1720-318 is very likely a new XN and $\mathrm{BHC}$, possibly located in the galactic bulge.

Although there is little doubt about the origin of the soft thermal component and its modelling, the interpretation of the high-energy tail and its connection to the spectral states remain rather controversial. In the HSS, most of the X-rays are radiated by the accretion disc which is supposed to extend down very close to the BH horizon. The standard Shakura \& Sunyaev (1973) $\alpha$-disc, however, cannot produce hard radiation (in either of the spectral states). In the LHS, the disc component is weak or absent and, when observed, the fitted temperature is very low and the inner radius very large. In this state, the accretion disc is supposed to be truncated at a large radius. The hard component is generally attributed to thermal comptonisation of the disc soft radiation by a hot corona (Sunyaev \& Titarchuk 1980; Titarchuk 1994) located above the disc or in the inner part of the system, around and very close to the BH. Thermal comptonisation models fit well the spectra during the LHS and indeed the comptonisation parameters derived for XTE J1720-318 are in good agreement with those usually observed in BH systems in LHS. However, the details of the geometry and of radiation mechanisms at work are still not understood; the processes which lead to the spectral transition and the possible role of non-thermal (synchrotron) radiation are still very uncertain. For example, one set of models which explain the above geometry and the comptonisation origin of the hard emission in LHS are those based on Advection Dominated Accretion Flows (ADAF). They are hot radiatively inefficient flows where most of the energy is advected into the BH (Esin et al. 1998). During LHS, the ADAF takes place between the truncated inner disc and the $\mathrm{BH}$ horizon, and gives rise to a hot optically thin plasma responsible for the thermal comptonisation of the disc photons.

However, in the recent years, it has become apparent that in LHS, the BHB become bright in radio and display a clear correlation between the $\mathrm{X}$ and radio luminosities 
(see Fender et al. 2003). Observations of XTE J1720-318 with the ATCA radio telescope have shown that this source was bright at radio wavelengths during the secondary outburst (Brocksopp et al. 2004, in preparation), when we clearly saw the source in the LHS. Simple ADAF models have difficulty to explain such correlations. Models where a compact jet at the base of the $\mathrm{BH}$ plays a major role in the physical processes of such systems have been proposed (Markoff et al. 2001). In jet models of BHB, the high-energy emission seen during the LHS is interpreted as synchrotron emission from the jets which extends from radio to hard X-ray, naturally explaining the correlations observed during the LHS.

Besides, the high energy tail observed in HSS or in the intermediate states is not fully understood. The observed steep power law (index $\sim 2.7$ ) without significance of a break could also be related to the presence of a nonthermal component in the accretion flow which has been proposed in the so-called hybrid thermal/non-thermal models (Zdziarski et al. 2001; Poutanen \& Coppi 1998). Alternatively, comptonisation on a population of (thermalised) electrons with bulk motion (e.g. Titarchuk et al. 1997; Laurent \& Titarchuk 1999) may be responsible for this component. The fit of XTE J1720-318 HSS spectrum we performed with the BMC model does provide parameters similar to those obtained in other BHB (Borozdin et al. 1999). However, the XTE J1720-318 spectrum is significant only up to about $80 \mathrm{keV}$ so we cannot test the predicted presence of a highenergy break expected at energies greater than $200 \mathrm{keV}$.

Thanks to the imaging capability and sensitivity of INTEGRAL, it has been possible to study a faint transient source in the galactic bulge, to detect a secondary outburst in hard X-rays, typical of XN, and a spectral transition confirming the probable $\mathrm{BH}$ nature of the object and to obtain a significant spectrum up to $200 \mathrm{keV}$. The detection and study of the other $\mathrm{XN}$ of the galactic bulge with INTEGRAL will possibly provide more data on this kind of objects and will thus improve our understanding of the physics of BHB.

Acknowledgements. M.C.B. thanks J. Paul and P. Ferrando for careful reading and commenting the manuscript. JR acknowledges financial support from the French Space Agency (CNES). We thank Brocksopp et al. for providing a preprint of their paper in preparation. We thank the ESA ISOC and MOC teams for they support in scheduling and operating the ToO observations of XTE J1720-318. The present work is based on observations with INTEGRAL, an ESA project with instruments and science data centre funded by ESA member states (especially the PI countries: Denmark, France, Germany, Italy, Switzerland, Spain, Czech Republic and Poland, and with the participation of Russia and the USA) and with XMM-Newton, an ESA science mission with instruments and contributions directly funded by ESA member states and the USA (NASA).

\section{References}

Arnaud, K. A. 1996, in Astronomical Data Analysis Software and Systems V, ed. G. H. Jacoby, \& J. Barnes (San Francisco: ASP), ASP Conf. Ser., 101, 17

Borozdin, K., Revnivtsev, M., Trudolyubov, S., et al. 1999, ApJ, 517, 367
Brocksopp, C., Corbel, S., Fender, R. P., et al. 2004, in preparation Courvoisier, T. J.-L., Walter, R., Beckmann, V., et al. 2003, A\&A, 411, L53

Ebisawa, K., Ogawa, M., Aoki, T., et al. 1994, PASJ, 41, 375

Esin, A. A., Narayan, R., Cui, W., et al. 1998, ApJ, 505, 854

Fender, R. P., Gallo, E., \& Jonker, P. G. 2003, MNRAS, 343, L99

Goldoni, P., Goldwurm, A., Kuulkers, E., et al. 2003, Atel, 153

Goldwurm, A., David, P., Foschini, L., et al. 2003, A\&A, 411, L223

Gonzalez-Riestra, R., Rodriguez-Pascual, P. M., Santo-Lleo, M., et al. 2003, IAUC, 8080

Gros, A., Goldwurm, A., Cadolle Bel, M., et al. 2003, A\&A, 411, L179

Guainazzi, M., XMM-Newton Science Analysis Workshop 2001, WA3

Knödlseder, J. 2004, 5th INTEGRAL Workshop, to be published

Laurent, P., \& Titarchuk, L. 1999, ApJ, 511, 289

Lebrun, F., Leray, J. P., Lavocat, P., et al. 2003, A\&A, 411, L141

Lund, N., Budtz-Jorgensen, C., Westergaard, N. J. et al. 2003, A\&A, 411, L231

McClintock, J. E., \& Remillard, R. E. 2003 [arXiv: astro-ph 0306213]

Markoff, S., Flacke, H., \& Fender, R. 2001, A\&A, 372, L25

Markwardt, C. B. 2003, Atel, 115

Mas-Hesse, J. M., Giménez, A., Culhane, J. L., et al. 2003, A\&A, 411, L261

Miller, J. M., Fabian, A. C., \& Wijnand, R. 2002, ApJ, 570, L69

Mitsuda, K., Inoue, H., Koyama, K., et al. 1984, PASJ, 36, 741

Nagata, T., Kato, D., Baba, D., et al. 2003, PASJ, to appear [arXiv: astro-ph/0312012]

O'Brien, K., Clarke, F., Fender, R. P., et al. 2003, Atel, 117

Parmar, A. N., Kuulkers, E., Oosterbroek, T., et al. 2003, A\&A, 411, L421

Poutanen, J., \& Coppi, P. 1998, Phys. Scr., 77, 57 [arXiv: astro-ph/9711316]

Poutanen, J., \& Svensson, R. 1996, ApJ, 410, 249

Remillard, R. E., Levine, A. M., Morgan, E. H., et al. 2003, IAUC, 8050

Revnivtsev, M. 2003, A\&A, 410, 865

Rodriguez, J., Corbel, S., \& Tomsick, J. A. 2003, ApJ, 595, 1032

Rupen, M. P., Brocksopp, C., Mioduszewski, A. J., et al. 2003, IAUC, 8054

Shakura, N. I., \& Sunyaev, R. A. 1973, A\&A, 24, 373

Shrader, C., \& Titarchuk, L. 1999, ApJ, 521, L21

Sobczak, G. J., McClintock, J. E., \& Remillard, R. R. 1999, ApJ, 520, 776

Sobczak, G. J., McClintock, J. E., \& Remillard, R. R. 2000, ApJ, 544, 993

Skinner, G., \& Connell, P. 2003, A\&A, 411, L123

Sunyaev, R. A., \& Titarchuk, L. 1980, A\&A, 86, 21

Tanaka, Y., \& Lewin, W. H. G. 1995, in X-ray Binaries, ed. W. H. G. Lewin, J. van Paradijs, \& E. P. J. van deu Heuvel (Cambridge University Press), 126

Tanaka, Y., \& Shibazaki, N. 1996, ARA\&A, 34, 607

Titarchuk, L. 1994, ApJ, 434, 570

Titarchuk, L., Mastichiadis, A., \& Kylafis, N. D. 1997, ApJ, 487, 834

Ubertini, P., Lebrun, F., Di Cocco, G., et al. 2003, A\&A, 411, L131

Winkler, C. 2001, in Exploring the Gamma-Ray Universe, Proceedings of the Fourth INTEGRAL Workshop, ESA SP-459, 471

Winkler, C., Courvoisier, T. J.-L., Di Cocco, G., et al. 2003, A\&A, 411, L1

Vedrenne, G., Roques, J.-P., Schönfelder, V., et al. 2003, A\&A, 411, L63

Zdziarski, A. A., Grove, J. E., Poutanen, J., et al. 2001, ApJ, 554, L45 